\title{
FATORES QUALITATIVOS DA PONTA DE ENERGIA HIDRÁULICA ADGA 110015 PARA PULVERIZAÇÃO AGRÍCOLA
}

\author{
MARCELO C. FERREIRA ${ }^{1}$, GLAUBERTO M. COSTA ${ }^{2}$, ADRIANA R. SILVA ${ }^{3}$, \\ SÔNIA R. A. TAGLIARI ${ }^{4}$
}

\begin{abstract}
RESUMO: Este trabalho teve o objetivo de caracterizar o diâmetro e a uniformidade das gotas e o perfil de distribuição volumétrica da ponta denominada antideriva de grande ângulo (ADGA) 110015, nas pressões de 207 e $310 \mathrm{kPa}$, para determinar o seu espaçamento na barra de pulverização. Os perfis de distribuição volumétrica para a altura de $50 \mathrm{~cm}$ foram avaliados em mesa de deposição. A partir dos perfis de distribuição, simulou-se o padrão de deposição ao longo da barra de pulverização. O espectro do diâmetro de gotas foi determinado em analisador de tamanho de partículas por difração laser. Pode-se concluir que a ponta ADGA 110015 apresentou perfil de distribuição do jato simétrico nas pressões de trabalho de 207 e $310 \mathrm{kPa}$. Na maior pressão, pode-se operar com menor altura da barra ou com as pontas mais espaçadas entre si, pois o maior ângulo de aspersão do jato resultou em diminuição do coeficiente de variação. Entretanto, o aumento da pressão proporcionou redução significativa no diâmetro das gotas, aumentando o potencial de cobertura do alvo, a suscetibilidade à deriva e à evaporação.
\end{abstract}

PALAVRAS-CHAVE: bico, padrão de deposição, tamanho de gotas.

\section{QUALITATIVE FACTORS FOR FLAT FAN HYDRAULIC NOZZLES ADGA 110015 IN AGRICULTURAL SPRAYING}

\begin{abstract}
The aim of this work was to characterize the diameter and uniformity of droplets and volumetric distribution profile for no-drift and great angle nozzle (ADGA) 110015, at 207 and $310 \mathrm{kPa}$ of pressure, to determine its spacing in the spray boom. The spray deposition was simulated from the distribution profiles. The droplet diameter spectrum was determined by laser diffraction. ADGA 110015 nozzle presented a symmetric volumetric distribution profile at 207 and $310 \mathrm{kPa}$. For this pressure, it is possible to operate with smaller height of the spray boom or with the nozzles more spaced to each other, because the largest angle of the spray resulted in a decrease of the variation coefficient. However, concerning to the drop diameter, a significant reduction was observed, increasing the potential of covering of the target and the susceptibility to drift and evaporation.
\end{abstract}

KEYWORDS: nozzle, deposition pattern, droplet size.

\section{INTRODUÇÃO}

A ocorrência de pragas, doenças e plantas infestantes no agroecossistema em importantes culturas (soja, milho, trigo, arroz, algodão, café) tem causado perdas de produtividade maiores que $30 \%$ do potencial produtivo, na média mundial, mesmo com práticas de controle (ECPA, 1992a, 1992b). Equipes multidisciplinares de pesquisadores têm trabalhado para compreender os problemas fitossanitários e encontrar formas de minimizá-los (MATTHEWS, 2000). Uma das formas mais utilizadas para salvaguardar as culturas das pragas tem sido a aplicação de produtos fitossanitários, especialmente sob a forma de pulverização.

\footnotetext{
${ }^{1}$ Engo Agrônomo, Prof. Dr., Departamento de Fitossanidade, UNESP, Jaboticabal - SP, Fone: (0XX16) 3203-2641, mdacosta@fcav.unesp.br

${ }^{2}$ Eng $^{-}$Agrônomo, Mestrando, Departamento de Defesa Fitossanitária, UNESP, Jaboticabal - SP

${ }^{3}$ Eng $^{\mathbf{a}}$ Agrônoma, Doutoranda, Departamento de Produção Vegetal, UNESP, Jaboticabal - SP

${ }^{4}$ Bióloga, Mestranda, Departamento de Entomologia Agrícola, UNESP, Jaboticabal - SP

Recebido pelo Conselho Editorial em: 16-8-2006
}

Aprovado pelo Conselho Editorial em: 25-4-2007 
A tecnologia de aplicação de produtos fitossanitários consiste na utilização de conhecimentos científicos para a correta colocação do produto biologicamente ativo no alvo, de forma econômica, na quantidade necessária e com o mínimo de contaminação de áreas não-alvo (MATUO, 1990). Para a pulverização, essa definição trata da utilização de gotas de tamanho adequado, sendo depositadas em quantidade suficiente na superfície do alvo para o controle do problema fitossanitário. Dessa forma, é imprescindível o uso da ponta de pulverização adequada, produzindo gotas com diâmetros que proporcionem o controle com a mínima quantidade de produto e a mínima contaminação do ecossistema (HIMEL, 1969), integrando-se ao conceito de tecnologia de aplicação. As gotas produzidas devem ter boa uniformidade de diâmetro, minimizando a percentagem de gotas menores que $100 \mu \mathrm{m}$, e que estão mais sujeitas à deriva, ou aquelas excessivamente grandes, que, após o impacto na superfície tratada, ricocheteiam e se fragmentam em gotas menores ou escorrem para o solo, devido ao uso de volume excessivo de calda (FERREIRA, 2003; CUNHA et al., 2003).

Sabendo-se dessas características, torna-se clara a necessidade de estratégias diferenciadas para cada tipo de aplicação, havendo ainda muito a se desenvolver e utilizar a respeito da formação, transporte e deposição das gotas, a fim de conseguir a correta colocação do produto no alvo. Perdas por escorrimento e/ou deriva superiores a $70 \%$ do volume aplicado já foram verificadas na cultura do tomate (CHAIM et al., 1999 a e 1999 b) e superiores a $50 \%$ na cultura de citros (MATUO, 1988).

Sendo a energia hidráulica a mais utilizada na realização da pulverização, é essencial conhecer o comportamento dos componentes envolvidos, sendo a ponta de pulverização a parte mais importante, por ser responsável pela formação e distribuição das gotas na área-alvo (MATUO, 1990). Por esse motivo, é imperativo conhecer suas características para oferecer aos usuários informações que permitam escolher o modelo mais adequado, com critério e segurança. As pontas antideriva de grande ângulo (ADGA), fabricadas no Brasil, são indicadas pelo fabricante para aplicações a baixas pressões sem alterações no ângulo do jato aspergido, produzindo gotas maiores, que resistem à deriva, adequando-se à definição de tecnologia de aplicação (MAGNOJET, 2006).

O espaçamento entre pontas na barra, a altura da barra em relação ao alvo, o ângulo do jato produzido pelas pontas e o diâmetro das gotas devem ser conhecidos para aplicação criteriosa. A uniformidade de distribuição pode ser calculada a partir da faixa de distribuição individual das pontas obtida em mesa de deposição, em laboratório. Assim sendo, o coeficiente de variação da distribuição volumétrica, para as diferentes distâncias entre pontas na barra de pulverização, é o parâmetro mais utilizado nessas situações (FAO, 1998). WOLF \& SMITH (1979) consideraram aceitáveis, para as condições do teste, coeficiente de variação de $15 \%$ para a aplicação de produtos sólidos. Segundo SMITH (1992), para boa distribuição de produto via líquida em campo, 15\% seria o coeficiente de variação máximo. Devido às variações inerentes às operações no campo, concluíram ser mais adequada a adoção de coeficiente de variação máximo de $10 \%$ nas aplicações via líquida, aumentando, assim, a qualidade da aplicação (PERECIN et al., 1994; PERECIN et al., 1998).

PERECIN et al. (1999) avaliaram diferentes pontas de pulverização por meio da vazão e do padrão de deposição em diferentes pressões e alturas do alvo. Observaram os melhores resultados para as pontas TF e XR; a ponta TK apresentou os piores resultados. BAUER \& RAETANO (2004b), avaliando o coeficiente de variação das pontas XR 11003 (200 e 300 kPa) e TXVK-4 (300 e $400 \mathrm{kPa}$ ) a 30; 40 e $50 \mathrm{~cm}$ do alvo, simulando sobreposição de $33 \%$ entre os jatos de calda, não observaram valores menores ou iguais a 7\%, sugerindo maiores estudos a esse respeito. Essas informações colaboram para que os usuários dessa tecnologia solicitem aos fabricantes o aprimoramento dos produtos para a disponibilização de peças com grau crescente de qualidade.

Este trabalho teve o objetivo de caracterizar a distribuição volumétrica, o diâmetro e a uniformidade de gotas produzidas pela ponta de pulverização hidráulica denominada antideriva de grande ângulo (ADGA) 110015, submetida às pressões de 207 e $310 \mathrm{kPa}$. 


\section{MATERIAL E MÉTODOS}

O trabalho foi realizado no Laboratório de Análise do Tamanho de Partículas (LAPAR), do Departamento de Fitossanidade da UNESP, Câmpus de Jaboticabal-SP, onde foram caracterizados o diâmetro e a uniformidade das gotas e o perfil de distribuição volumétrica da ponta MAGNO ADGA 110015 (ponta de cerâmica, jato plano de uso ampliado), nas pressões de 207 e $310 \mathrm{kPa}$.

\section{Distribuição volumétrica e espaçamento entre as pontas na barra de pulverização}

A determinação do perfil de distribuição volumétrica foi realizada em mesa de deposição composta por uma chapa de metal corrugada, formando 67 canaletas em "V", separadas de 2,5 cm entre si, totalizando $167,5 \mathrm{~cm}$ de largura.

Foram utilizados três exemplares de pontas ADGA 110015, instalados isoladamente no centro da mesa, de modo que o jato fosse lançado na posição vertical, a $50 \mathrm{~cm}$ de altura, segundo recomendações da FAO (1998). Após a instalação de cada ponta, iniciou-se a pulverização de uma calda contendo água com $0,1 \%$ do espalhante adesivo não-iônico (FAO, 1998) polioxietileno alquil fenol éter (Haiten), na pressão preestabelecida $\left(\mathrm{CO}_{2}\right.$ comprimido). Efetuou-se a medida direta do ângulo de aspersão do jato, com goniômetro analógico. Coletou-se o volume pulverizado durante $60 \mathrm{~s}$ em tubos de ensaio de $100 \mathrm{~mL}$, instalados sob as canaletas, com posterior quantificação em provetas de $100 \mathrm{~mL}$.

O perfil de distribuição volumétrica foi determinado com base na média dos volumes coletados nas três repetições em cada pressão. Esse perfil foi usado para calcular em planilha eletrônica (Microsoft Excel®) os padrões de distribuição volumétrica, simulando sobreposições do jato aplicado em diferentes espaçamentos entre pontas, ao longo da barra de pulverização. Os espaçamentos simulados variaram de 2,5 cm (valor correspondente à largura da canaleta coletora).

A uniformidade de distribuição ao longo da faixa aplicada foi avaliada por meio do coeficiente de variação (FAO, 1998), sendo utilizado o espaçamento-base de $50 \mathrm{~cm}$, recomendado pelo fabricante para a altura de $50 \mathrm{~cm}$ (MAGNOJET, 2006).

A simetria do perfil de distribuição do jato aspergido, indicativa da qualidade da ponta de pulverização, foi analisada utilizando o software GENES. Nesse programa, a simetria é avaliada pelo teste t de Student, tendo como hipótese da nulidade que os dados do perfil de distribuição possuem média igual a zero.

Estabeleceu-se equação de regressão dos coeficientes de variação, comparando-se as diferentes distâncias entre as pontas dentro das pressões de trabalho avaliadas. Os coeficientes de variação foram tomados como variáveis dependentes, e as distâncias entre pontas, na barra de pulverização, como variável independente.

\section{Determinação do diâmetro do espectro e da uniformidade das gotas}

O espectro de gotas produzido pela ponta ADGA 110015 foi determinado de forma direta em analisador de diâmetro de partículas por difração de raios laser (Mastersizer $S^{\circledR}$, versão 2.15). Nesse equipamento, uma unidade óptica determina o diâmetro das gotas do espectro pulverizado por meio do desvio de trajetória sofrido pelo laser ao atingi-las. Quanto menor a partícula, maior é o grau de difração que o raio de luz sofre (ETHERIDGE et al., 1999).

Conforme recomendações do fabricante, o equipamento foi ajustado para avaliar gotas de 0,5 a $900 \mu \mathrm{m}$ (lente $300 \mathrm{~mm}$ ). Um exaustor (tipo coifa) localizado sobre o equipamento, onde é pulverizado o jato para a determinação do espectro de gotas, retira as partículas que ficam suspensas no ar, podendo resultar em duplas leituras ou se depositar sobre a lente do aparelho, comprometendo a exatidão da análise.

Para manter a pressão constante, utilizou-se de ar comprimido controlado com regulador de pressão de precisão. Utilizou-se de oscilador para que o jato de calda atravessasse transversalmente 
o laser durante a leitura pelo aparelho. O tempo despendido pelo oscilador para mover-se de um extremo ao outro do jato aplicado foi calibrado para 3 segundos. Cada leitura do laser foi realizada com intervalo de 2 milissegundos, totalizando 500 leituras por segundo.

Foram realizadas seis repetições da leitura em cada pressão de trabalho avaliada, para a determinação dos seguintes parâmetros: diâmetro da gota, para o qual 10\% do volume pulverizado apresenta gotas de diâmetro inferior a ele $\left(\mathrm{Dv}_{0,1}\right)$; diâmetro mediano volumétrico ou o diâmetro da gota, para o qual 50\% do volume pulverizado apresenta gotas de diâmetro inferior a ele (DMV ou $\mathrm{Dv}_{0,5}$; ; diâmetro da gota, para o qual $90 \%$ do volume pulverizado apresenta gotas de diâmetro inferior a ele $\left(\mathrm{Dv}_{0,9}\right)$, e a uniformidade do espectro de gotas.

A uniformidade foi calculada pela amplitude relativa do espectro de gotas. A uniformidade máxima do jato é obtida quando a amplitude relativa se iguala a zero.

A amplitude relativa (AR) é dada por:

$$
\mathrm{AR}=\frac{\mathrm{Dv}_{0,9}-\mathrm{Dv}_{0,1}}{\mathrm{Dv}_{0,5}}
$$

em que,

AR - amplitude relativa ao diâmetro de gotas do jato aplicado;

$\mathrm{Dv}_{0,1}$ - diâmetro da gota, para o qual $10 \%$ do volume pulverizado apresenta gotas de diâmetro inferior a ele;

$\mathrm{Dv}_{0,5}$ - diâmetro da gota, para o qual $50 \%$ do volume pulverizado apresenta gotas de diâmetro inferior a ele, $\mathrm{e}$

$\mathrm{Dv}_{0,9}$ - diâmetro da gota, para o qual $90 \%$ do volume pulverizado apresenta gotas de diâmetro inferior a ele.

Os resultados de $\mathrm{Dv}_{0,1} ; \mathrm{Dv}_{0,5} ; \mathrm{Dv}_{0,9}$ e uniformidade de gotas produzidos pela ponta ADGA 110015 , nas pressões de 207 e $310 \mathrm{kPa}$, foram submetidos ao teste $\mathrm{F}$ para a análise de variância, e as médias foram comparadas pelo teste de Tukey $(\mathrm{P}<0,05)$.

\section{RESULTADOS E DISCUSSÃO}

\section{Distribuição volumétrica e espaçamento entre as pontas na barra de pulverização}

Os volumes coletados na mesa de deposição, nas pressões de 207 e $310 \mathrm{kPa}$, foram semelhantes entre si, apresentando padrão de deposição descontínuo, decrescendo do centro para as extremidades do jato pulverizado (Figura 1). A instalação, em barras de pulverização, de pontas que apresentam padrão descontínuo, implica necessidade de sobreposição consecutiva entre os jatos pulverizados (MATUO et al., 2001).

Apesar da semelhança entre os perfis de deposição quantificados na mesa de deposição, a pressão de trabalho influenciou no ângulo formado pelo jato de pulverização, ampliando a faixa de deposição da ponta e, conseqüentemente, a distância entre pontas na barra. Para a pressão de $207 \mathrm{kPa}$, o ângulo de abertura do jato foi de $100^{\circ}$, e as pontas apresentaram vazão média de $0,48 \mathrm{~L} \mathrm{~min}^{-1}$. Para a pressão de trabalho de $310 \mathrm{kPa}$, o ângulo foi de $110^{\circ} \mathrm{e}$ a vazão média de $0,59 \mathrm{~L} \mathrm{~min}^{-1}$.

Para o padrão de deposição do volume aplicado a $207 \mathrm{kPa}$ e $50 \mathrm{~cm}$ de distância entre pontas, o coeficiente de variação foi de $10,83 \%$. Já para a pressão de $310 \mathrm{kPa}$, o coeficiente de variação para a mesma distância entre pontas foi de $3,24 \%$. O comportamento desses dados está de acordo com aqueles obtidos por BAUER \& RAETANO (2004a), que observaram variação no perfil de deposição de 170 para $185 \mathrm{~cm}$, variando a pressão de 200 para $300 \mathrm{kPa}$, e coeficientes de variação do volume depositado menores que 10\% para a ponta de pulverização XR 11003.

Por meio da análise realizada pelo software GENES, concluiu-se que os perfis de distribuição volumétrica são simétricos $(\mathrm{P}<0,05)$, para as duas pressões avaliadas (Figura 1). 


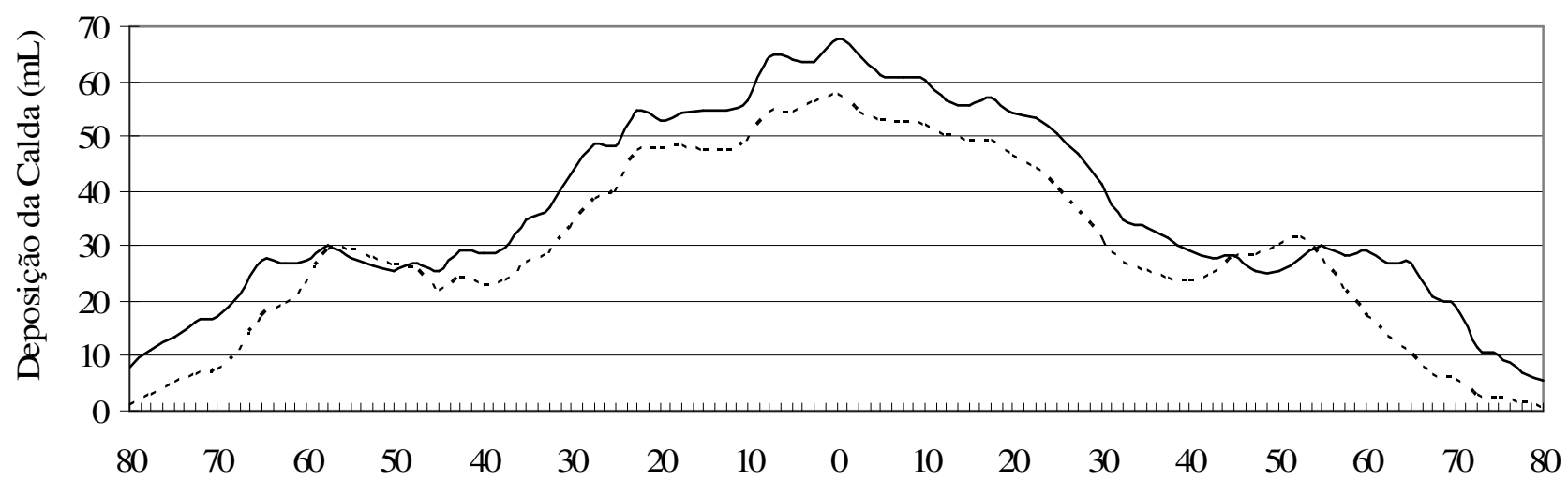

Distância em relação ao centro (0), em cm …. $207 \mathrm{kPa}-310 \mathrm{kPa}$

FIGURA 1. Perfis de distribuição volumétrica $(\mathrm{mL})$ do jato pulverizado pela ponta ADGA 110015 nas pressões de 207 e $310 \mathrm{kPa}$.

O coeficiente de determinação superior a 0,96 indica que ambos os lados do jato aspergido (esquerdo e direito) apresentaram simetria de deposição, para ambas as pressões avaliadas (Figura 2).
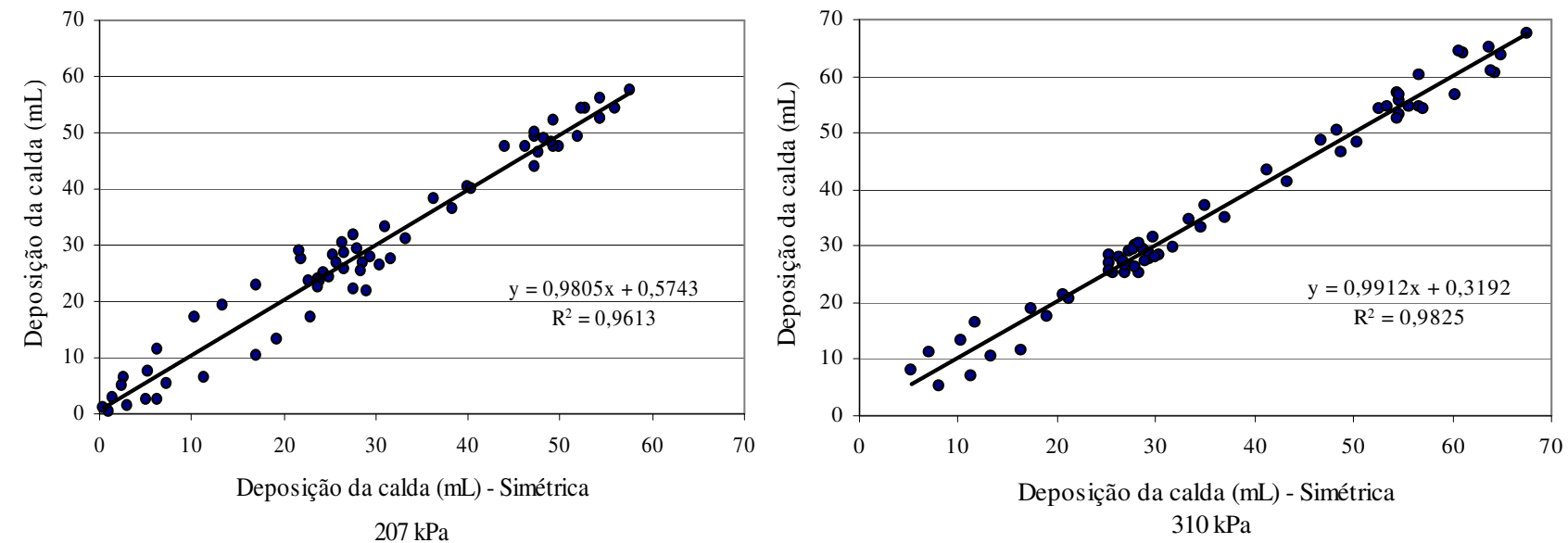

FIGURA 2. Equações de regressão linear e coeficientes de determinação para a simetria do perfil de deposição da calda proporcionada pela ponta de pulverização de jato plano ADGA 110015 , nas pressões de 207 e $310 \mathrm{kPa}$.

Na Figura 3, são apresentadas as curvas e equações de regressão linear para a sobreposição simulada dos coeficientes de variação do volume aplicado em função do espaçamento entre pontas na barra de pulverização. Os espaçamentos que implicaram coeficiente de variação de $10 \%$, foram iguais a 50,1 cm e 56,5 cm, para pressões de $207 \mathrm{kPa}$ e $310 \mathrm{kPa}$, respectivamente.

Avaliando o padrão de distribuição de pontas hidráulicas com ângulo de $110^{\circ} \mathrm{em}$ mesa de deposição e pressão de trabalho de $276 \mathrm{kPa}$, ETHERIDGE et al. (1999) observaram coeficientes de variação entre 12,1 e $27,2 \%$ para pontas distantes $51 \mathrm{~cm}$ entre si, a $41 \mathrm{~cm}$ da mesa. Os autores comentam que vazões que resultam em volumes de aplicação abaixo de $100 \mathrm{~L} \mathrm{ha}^{-1}$, podem ocasionar cobertura pobre e distribuição irregular, comprometendo a eficácia de controle. CUNHA \& RUAS (2006) observaram que, para pontas de jato plano duplo com indução de ar, o coeficiente de variação para a relação entre espaçamento e altura da barra em relação ao alvo, igual a 1,0, foi, respectivamente, de 10,$3 ; 4,2$ e $9,9 \%$ na pressão de $200 \mathrm{kPa}, 1,2 ; 11,5$ e 7,9\% na pressão de 
$300 \mathrm{kPa}$ e 6,6; 4,2 e 5,4\% na pressão de $400 \mathrm{kPa}$, para os modelos 110015; 11002 e 11003. Portanto, a maior vazão apresentou menores coeficientes de variação.

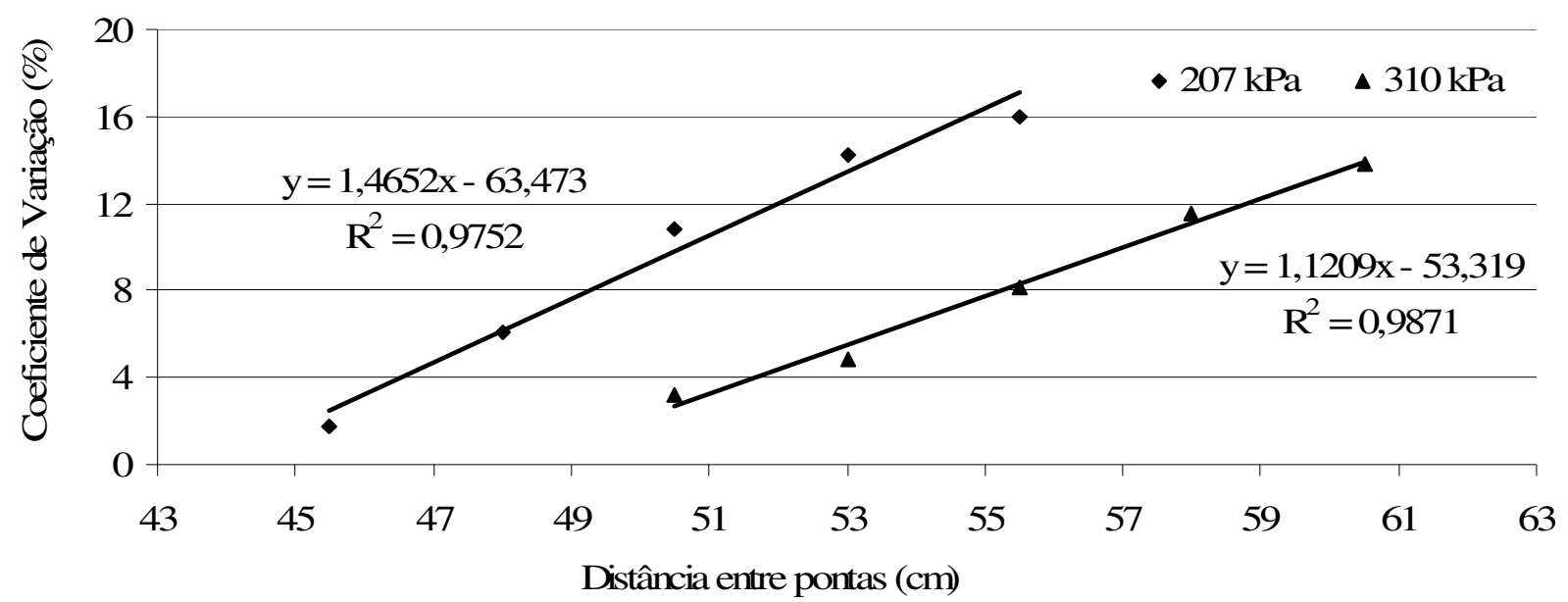

FIGURA 3. Coeficientes de variação do volume de calda aplicada em relação a distância entre pontas ADGA 110015, nas pressões de 207 e $310 \mathrm{kPa}$.

ETHERIDGE et al. (1999) sugerem que se aumente a altura da barra para diminuir o coeficiente de variação, ressaltando o aspecto da suscetibilidade à deriva das gotas quando em maior distância em relação ao alvo. Para BAUER \& RAETANO (2004a), a uniformidade de distribuição sofre maior influência do espaçamento entre as pontas do que da altura da barra. Os autores observaram coeficiente de variação menor que 7\% para pontas XR 8004, no espaçamento de $35 \mathrm{~cm}$ entre pontas para altura de $30 \mathrm{~cm}$, e no espaçamento de $60 \mathrm{~cm}$ para altura de $50 \mathrm{~cm}$.

Para a ponta ADGA 110015, avaliada neste trabalho, o ângulo de $110^{\circ}$ conseguido com a pressão de $310 \mathrm{kPa}$ permite a aproximação da barra em relação ao alvo. Entretanto, isso não é possível para a pressão de $207 \mathrm{kPa}$. Segundo o fabricante, a altura mínima recomendada é de $40 \mathrm{~cm}$ para pontas espaçadas de $50 \mathrm{~cm}$ entre si na barra de pulverização (MAGNOJET, 2006).

A uniformidade do jato é importante para a adequada distribuição do produto na área-alvo, aumentando a possibilidade de controle da praga visada. É importante ressaltar que as pontas de pulverização de jato plano devem ser instaladas de modo que o jato aspergido forme ângulo fixo em relação à barra (entre 6 e $10^{\circ}$ ) para evitar o choque entre jatos de calda adjacentes, o que compromete a uniformidade de deposição. Caso não haja simetria no jato aspergido, haverá coeficiente de variação se o ângulo entre o jato aspergido e a barra do pulverizador for positivo e outro se o ângulo for negativo.

O padrão observado para a ponta ADGA 110015, nas pressões de 207 e $310 \mathrm{kPa}$, pode ser considerado adequado para a instalação consecutiva dessas em barra de pulverização, na altura e no espaçamento avaliados neste trabalho. Isso está de acordo com o observado nos trabalhos de ETHERIDGE et al. (1999) e MATUO et al. (2001).

\section{Determinação do diâmetro e da uniformidade das gotas}

Houve diferença significativa para os diâmetros das gotas para os quais $10 \%\left(\mathrm{Dv}_{0,1}\right), 50 \%$ $\left(\mathrm{Dv}_{0,5}\right)$ e $90 \%\left(\mathrm{Dv}_{0,9}\right)$ dos volumes pulverizados apresentaram gotas de diâmetros inferiores a eles, respectivamente, nas pressões de trabalho avaliadas, e sob a pressão de $207 \mathrm{kPa}$, os diâmetros característicos foram maiores. A uniformidade do jato pulverizado não apresentou diferença significativa em ambas as pressões avaliadas (Tabela 1).

Segundo LEFEBVRE (1989), gotas de diâmetros próximos a $150 \mu \mathrm{m}$ são arrastadas com facilidade pelo vento e altamente suscetíveis à evaporação. ETHERIDGE et al. (1999) são ainda mais rigorosos, afirmam que gotas abaixo de $200 \mu \mathrm{m}$ são as mais suscetíveis à deriva. Deve-se considerar, entretanto, que a cobertura do alvo diminui com a formação de gotas maiores e também 
a possibilidade de abaixar a barra quando se aumenta a pressão, devido ao aumento do ângulo de aspersão do jato de pulverização.

TABELA 1. Diâmetro médio das gotas correspondentes aos volumes acumulados de $10\left(\mathrm{Dv}_{0,1}\right), 50$ $\left(\mathrm{Dv}_{0,5}\right)$ e $90 \%\left(\mathrm{Dv}_{0,9}\right)$ e uniformidade de gotas formadas pela ponta ADGA 110015, nas pressões de 207 e $310 \mathrm{kPa}$.

\begin{tabular}{ccccc}
\hline Pressão $(\mathrm{kPa})$ & $\mathrm{Dv}_{0,1}(\mu \mathrm{m})$ & $\mathrm{Dv}_{0,5}(\mu \mathrm{m})$ & $\mathrm{Dv}_{0,9}(\mu \mathrm{m})$ & Uniformidade \\
\hline 207 & $103,13 \mathrm{a}^{\mathrm{I}}$ & $200,20 \mathrm{a}$ & $431,61 \mathrm{a}$ & $0,50 \mathrm{a}$ \\
310 & $85,92 \mathrm{~b}$ & $168,56 \mathrm{~b}$ & $333,78 \mathrm{~b}$ & $0,46 \mathrm{a}$ \\
DMS & 5,21 & 6,73 & 19,75 & 0,03 \\
C.V. $(\%)$ & 3,80 & 4,50 & 4,30 & 5,20 \\
\hline
\end{tabular}

${ }^{\mathrm{T}}$ Médias seguidas de letras diferentes, na coluna, diferem entre si, pelo teste de Tukey $(\mathrm{P}<0,05)$.

\section{CONCLUSÕES}

A ponta avaliada caracterizou-se por boa distribuição volumétrica da calda, e o tamanho de gotas observado apresentou-se na faixa suscetível à deriva. As pressões avaliadas não alteraram a uniformidade do espectro de gotas.

\section{REFERÊNCIAS}

BAUER, F.C.; RAETANO, C.G. Distribuição volumétrica de calda produzida pelas pontas de pulverização XR, TP e TJ sob diferentes condições operacionais. Planta Daninha, Viçosa, v.22, n.2, p.275-84, 2004a.

BAUER, F.C., RAETANO, C.G. Perfis de distribuição volumétrica de pontas XR 11003 e TXVK4 em diferentes condições de pulverização. Engenharia Agrícola, Jaboticabal, v.24, n.2, p.364-73, $2004 b$.

CHAIM, A.; VALARINI, P.J.; OLIVEIRA, D.A.; MORSOLETO, R.V.; PIO, L.C. Avaliação de perdas de pulverização em culturas de feijão e tomate. Jaguariúna: Embrapa Meio Ambiente, 1999a. 10 p. (Boletim de Pesquisa, 2).

CHAIM, A.; VALARINI, P.J.; OLIVEIRA, D.A.; MORSOLETO, R.V.; PIO, L.C. Método para monitorar perdas na aplicação de agrotóxicos na cultura de tomate. Pesquisa Agropecuária Tropical, Brasília, v.35, n.5, p.741-7, 1999b.

CUNHA, J.P.A.R.; TEIXEIRA, M.M.; COURY, J.R.; FERREIRA, L.R. Avaliação de estratégias para a redução da deriva de agrotóxicos em pulverizações hidráulicas. Planta Daninha, Viçosa, v.21, n.10, p. 325-32, 2003.

CUNHA, J.P.A.R.; RUAS, R.A.A. Uniformidade de distribuição volumétrica de pontas de pulverização de jato plano duplo com indução de ar. Pesquisa Agropecuária Tropical, Brasília, v.36, n.1, p.61-6, 2006.

ECPA. EUROPEAN CROP PROTECTION ASSOCIATION. Cereals and plant protection. ECPA: Summary of the Cereal Chapters, 1992a. 16 p.

ECPA. EUROPEAN CROP PROTECTION ASSOCIATION. Non-cereal crops and plant protection. ECPA: Summary of the Non-Cereal Chapters, Covering Cotton, Potatoes, Soybean and Coffee, 1992b. 12 p.

ETHERIDGE, R.E.; WOMAC, A.R.; MUELLER, T.C. Characterization of the spray droplet spectra and patterns of four venturi-type drift reduction nozzles. Weed Technology, Lawrence, v.13, n.4, p.765-70, 1999.

FAO. FOOD AND AGRICULTURE ORGANIZATION OF THE UNITED NATIONS. Agricultural pesticide sprays. Rome: FAO, 1998. v.2, p.62. 
FERREIRA, M.C. Caracterização da cobertura de pulverização necessária para controle do ácaro Brevipalpus phoenicis (G., 1939) em citros. 2003. 64 f. Tese (Doutorado em Produção Vegetal) - Universidade Estadual Paulista, Faculdade de Ciências Agrárias e Veterinárias, Jaboticabal, 2003.

HIMEL, C.M. The optimum size for insecticide spray droplets. Journal of Economic Entomology. Lanham, v.62, n.4, p.919-25, 1969.

LEFEBVRE, A. H. Atomization and sprays. New York: Hemisphere Publishing Corporation, 1989. $421 \mathrm{p}$.

MAGNOJET. Produtos: ADGA, características. Disponível em: <http://www.magnojet.com.br> Acesso em: 16 julho 2006.

MATTHEWS, G.A. Pesticide application methods. London: Longman, 2000. 448 p.

MATUO, T. Desenvolvimento de um pulverizador intermitente operado fotoeletricamente para tratamento de pomares de citros. 1988. 167 f. Tese (Livre-Docência) - Universidade Estadual Paulista, Faculdade de Ciências Agrárias e Veterinárias, Jaboticabal, 1988.

MATUO, T. Técnicas de aplicação de defensivos agrícolas. Jaboticabal: FUNEP, 1990. 140 p.

MATUO, T.; PIO, L.C.; RAMOS, H.H.; FERREIRA, L.R. Tecnologia de aplicação e equipamentos. In: CURSO DE ESPECIALIZAÇÃO POR TUTORIA A DISTÂNCIA PROTEÇÃO DE PLANTAS: Módulo 02: 2.2, 2001, Brasília. 71 p.

PERECIN, D.; PERESSIN, V.A.; MATUO, T.; BRAZ, B.A.; PIO, L.C. Padrões de distribuição obtidos com bicos TF-VS4, TJ60-11006 e TQ15006 em mesa de prova. Pesquisa Agropecuária Tropical, Viçosa, v.33, n.2, p.175-82, 1998.

PERECIN, D.; PERESSIN, V.A.; MATUO, T.; BARBOSA, J.C.; PIO, L.C.; BRAZ, B.A. Padrões de distribuição obtidos com bicos Twinjet, em função da altura e do espaçamento entre bicos. Engenharia Agrícola, Campinas, v.14, p.19-30, 1994.

PERECIN, D.; PERESSIN, V.A.; MATUO, T.; BRAZ, B.A.; PIO, L.C. Avaliação do desempenho de bicos para aplicação de herbicidas. Planta Daninha, Viçosa, v.17, n.1, p.83-94, 1999.

SMITH, D.B. Uniformity and recovery of broadcast sprays using fan nozzles. Transactions of the $A S A E$, St. Joseph, v.35, n.1, p.39-44, 1992.

WOLF, D.D.; SMITH, E.F. Uniformity of seed and fertilizer distribution with a hand operated spinning spreader. Transactions of the ASAE, St. Joseph, v.22, n.16, p.761-2, 1979. 